\title{
Biomechanical Comparison of Polymeric Spinal Cages Using Ct Based Finite Element Method
}

\author{
Muhammad Hilmi Jalil ${ }^{1}{ }^{2}$, Muhammad Hazli Mazlan³, Mitsugu Todo ${ }^{4}$ \\ ${ }^{1}$ Interdisciplinary Graduate School of Engineering Sciences, Kyushu University. 6-1 Kasuga Koen, Kasuga, \\ Fukuoka, 816-8580, Japan. \\ 2 Universiti Malaysia Pahang,Pahang, Malaysia. \\ 3 Universiti Tun Hussein Onn Malaysia, Malaysia. \\ ${ }^{4}$ Research Institute of Applied Mechanics, Kyushu University, Japan. \\ * Corresponding author. Email: hilmijalil99@gmail.com \\ Manuscript submitted October 27, 2016; accepted January 18, 2017. \\ doi: 10.17706/ijbbb.2017.7.2.110-117
}

\begin{abstract}
Interbody fusion devices are gaining acceptance as a treatment method of mainly for disc degeneration diseases and other medical conditions. Posterior lumbar interbody fusion (PLIF) cage is used in the procedure to maintain stability and promote fusion between vertebrae. Poly lactic acid (PLA) is assumed to be the alternative material which could provide cheaper material and lower production cost. However, these implants often cause subsidence failure at the endplate, resulting in injury risk and mechanical instability during fusion. In this study, the stress behavior of PLIF cage made by two different materials, Polyether ether ketone (PEEK) and PLA; was studied using finite element method (FEM). By implementing bilateral cages between vertebral bone L4 and L5, and conducting 6 different motion activities onto the model, the stress distribution of L4-L5, and cage bodies was predicted. Simulation results predicted that the cage subsidence occurred at both materials, with an overall of higher cage-endplate stresses for PEEK, in comparison to PLA and controlled configurations. In addition, the stress distribution in PLA cage was better and the maximum von Mises stress was approximately 3 times lower than PEEK cage. Further investigation of PLA cage's mechanical properties should be done experimentally to determine the accuracy and reliability of the simulation.
\end{abstract}

Key words: Interbody fusion, PLA, spinal cage, subsidence.

\section{Introduction}

Spinal fusion has been a widely applied surgical procedure to treat spinal disc degeneration diseases that was due to the degeneration of annulus fibrosus. It is reported that in 2012 , the spinal fusion procedures made up to $5.7 \%$ of total operating room procedures performed in the United State (US) and it ranked at $5^{\text {th }}$ from 15 other procedures. In addition, in between 2003 and 2012, the average annual percentage change of spinal fusion procedures was increasing 3.1\% each years [1]. Posterior lumbar interbody fusion (PLIF) cage was mainly manufactured using Titanium (Ti) and Polyether ether ketone (PEEK). Although many impressive clinical results yielded in Ti cage's trials, excessive material and production cost has made the cost-effectiveness of using Ti arguable [2], [3]. Either Ti or PEEK, same problem is reported on the cage subsidence failure and stress shielding at cage-endplate interface that might lead to fracture risk [4]. Metal cage was often failed to stimulate bone tissue fusion and remodeling by effectively transferring loads [5], [6]. Usage of biodegradable polymer; e.g. poly lactic acid (PLA) has gained growing consideration due to its 
degradability, cheaper fabrication cost and similar mechanical properties to those of the vertebral bone [5], [7]. In spite of these advantages, PLA's strength level is notably inferior to Ti and PEEK cage, and it is difficult to define proper degradation rate that can ensures sufficient support during the healing and fusion process of the bone [8]. The advancement in fabrication technology has gave birth to 3D rapid prototyping, which allowed the consumer for cheaper and more complex cage design using PLA [9], [10]. In order to exploit these modern fabrication technique, it is important to understand the effect of different polymer materials used as PLIF cage to the stress behavior of implanted vertebral bone. Finite element analysis (FEA) method appears to be the most impressive tool for the investigation of the mechanics of bone and cage structures [11].

In this study, FEA was used to calculate the stress distribution on the lumbar vertebrae and the spinal cage with PEEK and PLA as the cage's built material. A finite element (FE) model without any cage and posterior instrumentation (PI) implanted was used as the control model. This study objectives is to quantitatively analyze and compare the biomechanical effect of using two different biomaterials, i.e., PEEK and PLA as the cage's base material. The FE analysis should give enlightenment on the cage subsidence effect in the PLIF procedure and give insight to the stress distribution of the vertebrae and cage altogether.

\section{Material and Method}

\subsection{FE Modelling}

FEA was applied in this study to evaluate the stress distribution and the cage subsidence phenomenon in the constructed lumbar vertebra. Concisely, thin-sliced computed tomography (CT) images were obtained from the fourth to five lumbar vertebrae, known as L4 and L5, respectively. The CT scan images were taken from a healthy 29-years old Japanese male (78 kg weight and $176 \mathrm{~cm}$ height) with a written informed consent permission received prior to the research. The FE model of L4 and L5 vertebrae was built using the CT images via the bone modelling function in the MECHANICAL FINDER ${ }^{\mathrm{TM}}$ software (Research Center of Computational Mechanics Co. Ltd. Japan). The anatomical structure of L4-L5 was obtained by utilizing the functions in the software and by extracting the region of interests (ROI) based on the extracted bone edges. L4 and L5 vertebral bodies were defined as cancellous bone core encased by a $0.4 \mathrm{~mm}$ thick cortical bone. Next, L4-L5 which consist of the cancellous bone, intervertebral discs and facet joint cartilage, were configured as solid tetrahedral elements of $1.0 \mathrm{~mm}$ in size; while the cortical bone was modelled with 1.0 mm linear shell triangular elements. The model contained approximately 1286000 solid elements and 91000 shell elements.

The mechanical properties of the bone model was calculated using the Hounsfield Unit (HU) values, where the bone density of each tetrahedral element was defined as the average number of HU units inside each of the corresponded elements. Utilizing the relationship reported by Keyak [12], the value of Young's modulus, yield stress and Poisson's ratio were obtained for the heterogeneous bone model as shown in Table 1. Poisson's ratio for the facet joints and intervertebral discs were defined as 0.45 and 0.2 respectively. While the Young's modulus were set at $8.4 \mathrm{MPa}$ and $11 \mathrm{MPa}$ for the facet joint and discs, correspondingly [13]. Fig. 1 showed the solid and meshed model of the simulated L4-L5 vertebral bone attached with PI.

A commercially available PLIF cage, TELAMON PEEK ${ }^{\mathrm{TM}}$ (Medtronic Sofamor Danek, Memphis, TN) with a dimension of $26 \mathrm{~mm}$ in length, $8 \mathrm{~mm}$ in height and $10 \mathrm{~mm}$ in width was used in this study. A conventional bilateral mode configuration was applied to simulate standard PLIF procedure in the L4-L5. PLIF surgery procedure was simulated in this study by trimming the facet joints in order to allow the application of the posterior pedicle screw fixation. This screw-rods posterior instrumentation (PI) included two rods and two screws with the diameter of $6.2 \mathrm{~mm}$ (rods and screws) and the screw length of $51.8 \mathrm{~mm}$. Several part of the discs including the nucleus pulposus and the annulus fibrosus were subtracted to give space for bilateral cages insertion. In this study, the material properties of the spinal cage was defined as PEEK and PLA, as 
shown in Table 2. PI components was defined as Ti-6Al-4V, and the mechanical properties was shown in the same table. All cages and PI components were modelled with the same tetrahedral elements, with minimum mesh size of $1.0 \mathrm{~mm}$. The amount of bilateral spinal cage model's solid element was approximately 108700 elements.

Table 1. Material Properties of Bone Model

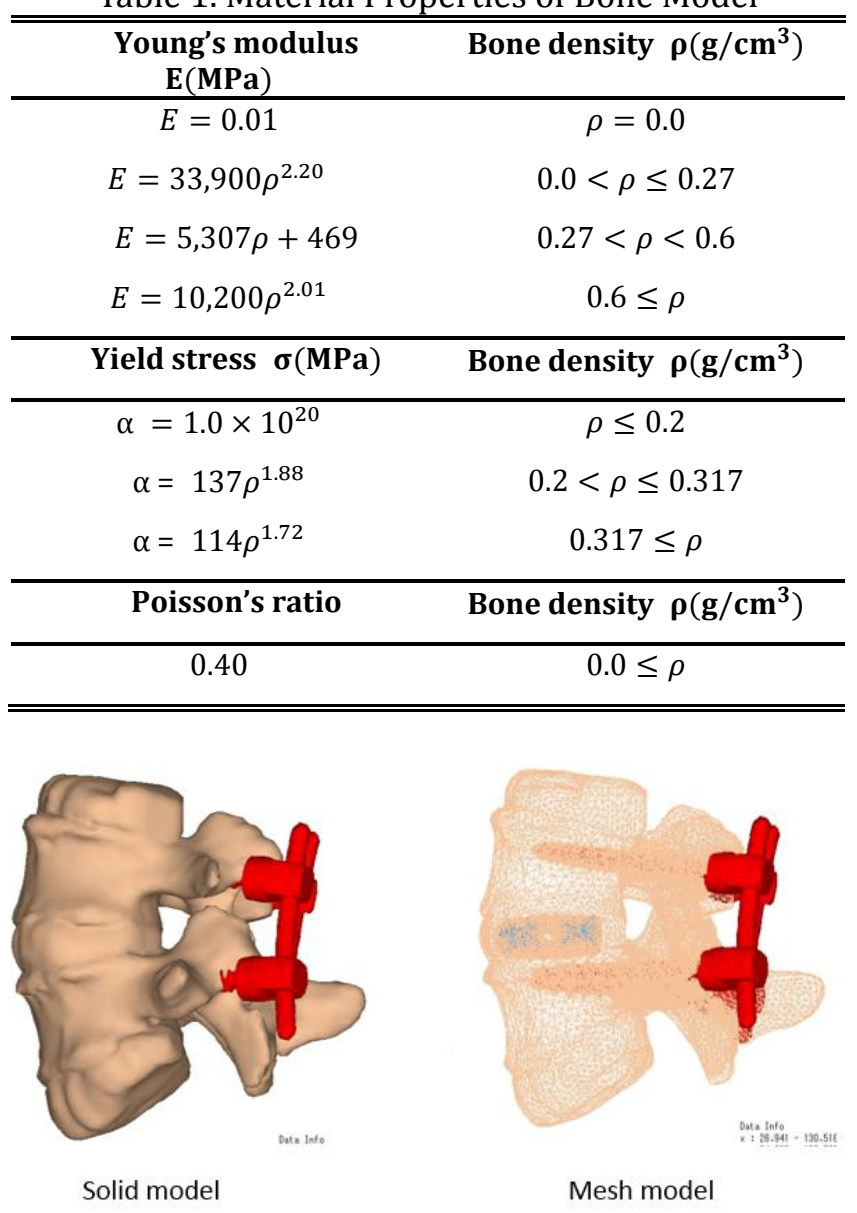

Fig. 1. Simulated L4-L5 bone.

Table 2. Material Properties of Spinal Cage

\begin{tabular}{cccccc}
\hline \hline Material & $\begin{array}{c}\text { Young`s } \\
\text { modulus (MPa) }\end{array}$ & $\begin{array}{c}\text { Critical } \\
\text { stress }(\mathbf{M P a})\end{array}$ & $\begin{array}{c}\text { Density } \\
\left.\mathbf{( k g} / \mathbf{m m}^{3}\right)\end{array}$ & $\begin{array}{c}\text { Poisson } \\
\text { ratio }\end{array}$ & $\begin{array}{c}\text { Stress } \\
\text { relaxation }\end{array}$ \\
\hline PEEK & $3.620 \times 10^{3}$ & 130 & $1.32 \times 10^{-6}$ & 0.39 & 0.1 \\
PLA & $1.459 \times 10^{3}$ & 231.2 & $1.28 \times 10^{-6}$ & 0.4 & 0.1 \\
Ti-6Al-4V & $1.14 \times 10^{5}$ & 970 & $4.43 \times 10^{-6}$ & 0.34 & 0.1 \\
\hline \hline
\end{tabular}

\subsection{Analysis}

Loading and boundary condition were applied in this study to represent the load during normal physiological activities of the spine. The FE models were loaded with two compressive loads of $10 \mathrm{kN}$ and $1000 \mathrm{~N}$, and four rotational loads, namely as flexion, extension, lateral bending and axial rotation. As shown in the Fig. 2, the loads were applied on the superior surface of L4, where red nodes were represented. All nodes (referred as blue nodes in Fig. 2) on the inferior surface of L5 were constrained in every degrees of freedom. Maximal Drucker-Prager stress from PEEK, PLA, and no cage model configuration; together with different loading conditions were compared to evaluate the stress behavior of the spinal bone. Bone 
fracture analysis was also evaluated on L4-L5 in order to predict and to find the distribution of bone fracture risk using the nonlinear Newton-Raphson method [14]. Observation on the predicted maximum von Mises stress distribution in the cage bodies was done to understand the effect of different materials and loading activities on the cage itself [15].

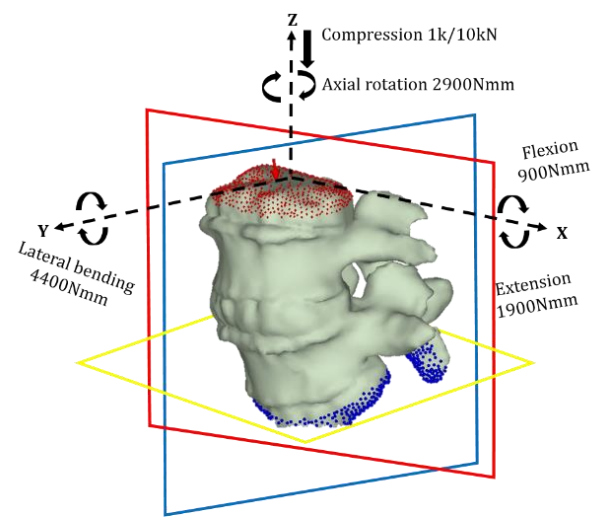

Fig. 2. Loading and boundary condition of FE model.

\section{Result and Discussion}

Based on the FEA, a cross section of L4-L5 images of stress distribution was taken to understand the stress behavior and the cage subsidence effect on both vertebral bones. Fig. 3 summarizes the Drucker-Prager stress distribution of different cage material configuration under each loading condition. Concentrated stresses were observed at the proximity of cage endplate; i.e., the inferior surface of L4 and superior surface of L5, and it was in agreement with previous FEM study [16]. The stresses distortion at the cage-endplate interface was highly caused by the difference in stiffness of the cancellous bone and the spinal cages. These might followed with cage subsidence, which occurred when the PLIF cages subsided into the vertebral body and induced kyphotic deformity [17]. High stress concentrated also occurred at the facet joints and spinous process which connected L4 and L5. Controlled-none cage result showed significant stress concentration in comparison with PEEK and PLA, especially for compression $10 \mathrm{kN}$, flexion, extension and axial rotation's loading. These might be the result of installing PI together with PLIF cage into the bone model. PI which is generally applied to act as an auxiliary support system for the bone fusion process, was actually helping to decrease and stabilize the stress distribution inside the bone model. The functionality of PI was approved to decrease the stress distortion of cage-endplate interface and facet joints by at least half [18].

The Drucker-Prager stress was adopted in this evaluation as suggested by Bessho [19] , because this stress criterion was suitable for brittle material such as bone, and it also considered the contribution of hydrostatic stress. 20 areas of interest (ROI) were selected from the models, by extracting 10 different slices from the superior to the inferior part of each vertebral bone model. Each slices thickness was $2 \mathrm{~mm}$ and the distance between adjacent slices was $3 \mathrm{~mm}$. Fig. 4 represented the maximal Drucker-Prager stresses distribution of each ROI of L4 and L5. From the figure, PEEK and PLA configuration showed similar stress profile between all motion activities. At cage-endplate interface (ROI 10 and 11), a sudden increase in stress values were observed at every loading of PEEK and PLA. Excluding the lateral bending loading, where several spikes appeared; these stress spikes showed clearly that cage application had caused subsidence phenomenon inside the bone structure. As comparison, none cage setting exhibited much lower and uniform pattern at the compression of $10 \mathrm{kN}$, compression of $1000 \mathrm{~N}$, extension and axial rotation. 


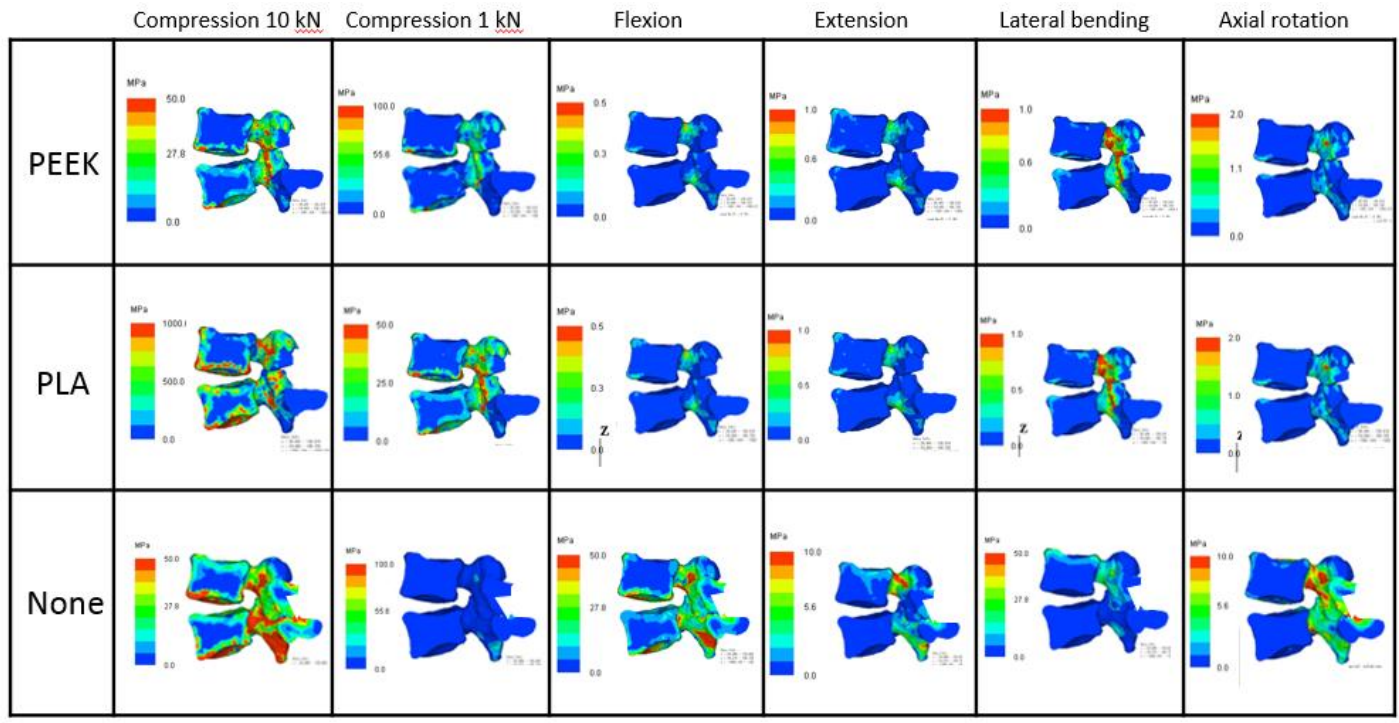

Fig. 3. Drucker-Prager stress distributions for PEEK, PLA and None cages configuration.
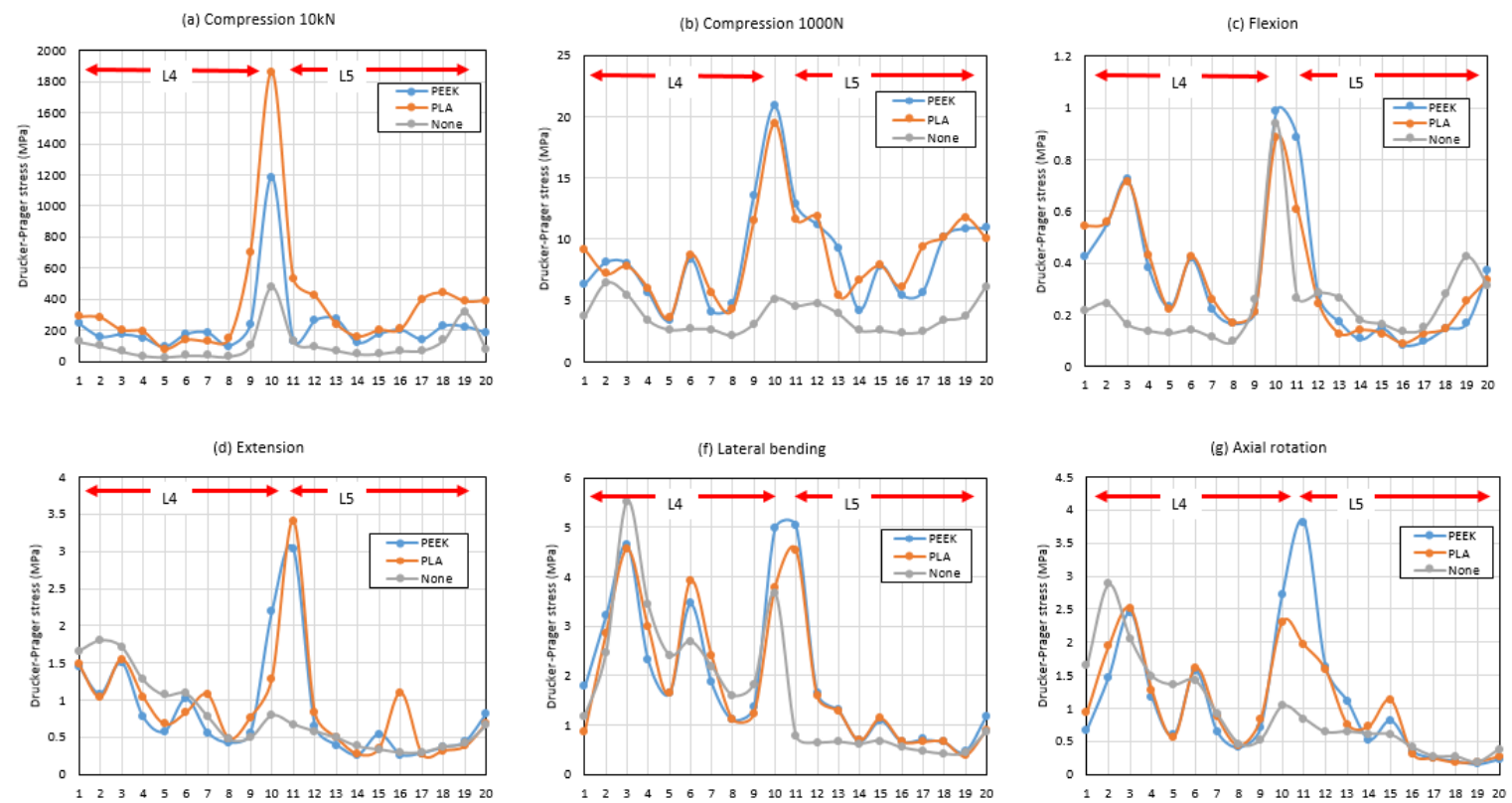

Fig. 4. Maximal Drucker-Prager stress distribution of (a) compression 10 kN, (b) compression 1000 N, (c) flexion, (d) extension, (f) lateral bending and (g) axial rotation.

Based on Fig. 4, maximal Drucker-Prager stresses of the cage-endplate interface were plotted and showed in Fig. 5. Under extreme compression of $10 \mathrm{kN}$, Drucker-Prager stresses for PEEK, PLA and None were at 1186.5 MPa, 1863.2 MPa and 480.6 MPa, respectively. For another loading of compression $1000 \mathrm{~N}$, flexion, extension, lateral bending and axial rotation; the stresses for PEEK cage were $20.9 \mathrm{MPa}, 1 \mathrm{MPa}, 4.9 \mathrm{MPa}, 7.9$ $\mathrm{MPa}$ and 5.2 MPa; respectively. The Drucker-Prager stresses for PLA cage were $19.5 \mathrm{MPa}, 1 \mathrm{MPa}, 3 \mathrm{MPa}, 4.3$ $\mathrm{MPa}$, and $5.2 \mathrm{MPa}$ for compression $1000 \mathrm{~N}$, flexion, extension, lateral bending and axial rotation. Similarly, stresses for None cage configuration were $4.5 \mathrm{MPa}$, $1 \mathrm{MPa}$, 2.9 MPa, 4.8 $\mathrm{MPa}$ and $3.7 \mathrm{MPa}$. Under extreme compression of $10 \mathrm{kN}$, PLA was predicted to have higher stress value in comparison to PEEK. Similar Drucker-Prager stresses value were observed between PEEK and PLA for flexion and axial rotation. PLA showed lower stress value for compression of $1000 \mathrm{~N}$, extension and lateral bending. Generally comparing with None cage configuration, PEEK and PLA showed relative difference value percentage from $1 \%$ to $365 \%$, 
and from $-10 \%$ to $333 \%$, respectively.

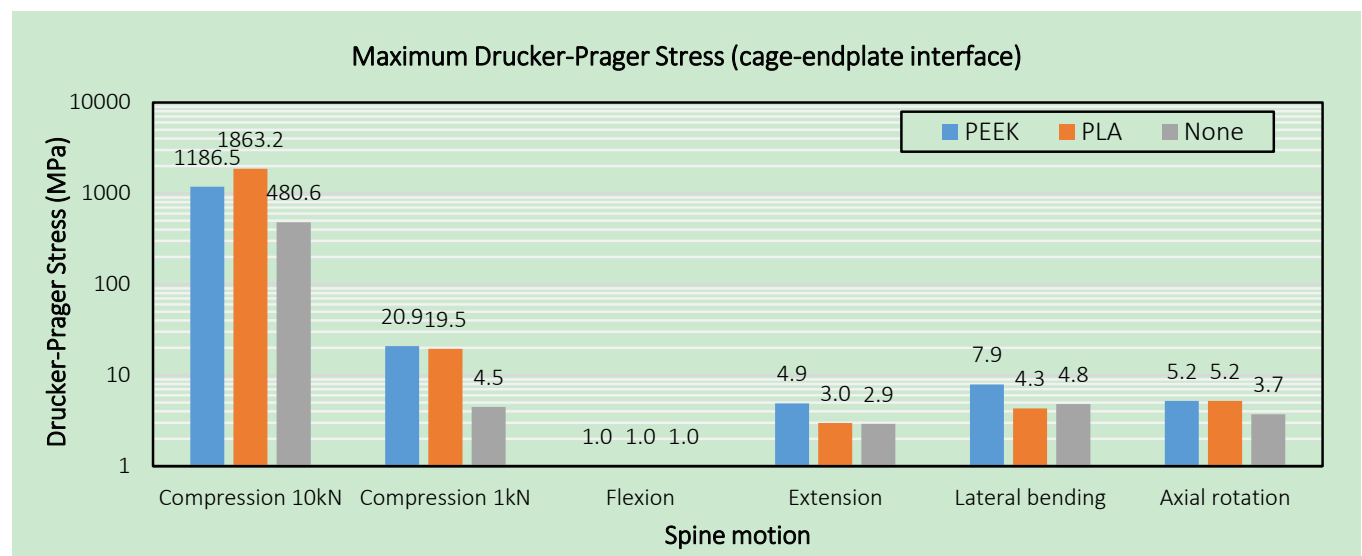

Fig. 5. Maximal Drucker-Prager stress at cage-endplate interface.

The largest relative difference of Drucker-Prager stresses showed by PEEK and PLA was under the compression of $1000 \mathrm{~N}$, which is at $365 \%$ and $333 \%$, respectively. Therefore, von Mises stress distribution at cage bodies of PEEK and PLA, was observed to help us understand the load transfer mechanism between the vertebral endplate and implant bodies. The von Mises stress value was often used because it considered all of the normal and shear stress components acting in the material [15]. From Fig. 6, an extensive observation was the spotty stress distribution at the anterior superior edges and at the beneath side of inferior surface of each PEEK and PLA implants. The cage implant might had failed to distribute the compression load from the superior endplate surface to the beneath surface evenly, and it tend to deform the vertebral endplate by having the posterior and inferior edges of the implants to mainly support it.

(a) PEEK

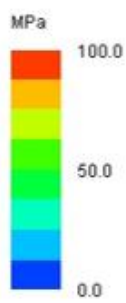

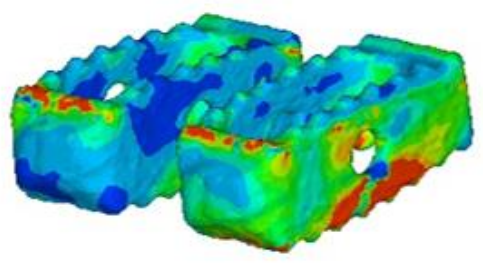

(b) PLA

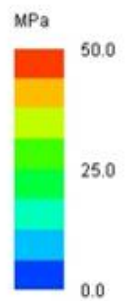

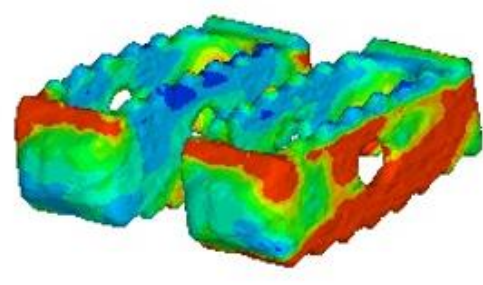

Fig. 6. Von Misses stress distribution on cage body of compression $1000 \mathrm{~N}$ loading.

For compressive loading of $1000 \mathrm{~N}$, the nominal contact stress could be calculated as $\sigma=F / A=$ $5.02 \mathrm{MPa}$ (assuming full-face contact between cages and bone, where surface area of cage, $A=99.6 \mathrm{~mm}^{2}$ and $F=500 \mathrm{~N}$ per cage). Comparing with the predicted maximum von Mises at cage bodies for PEEK and PLA which were 889.8 MPa and 248.1 MPa, respectively; the predicted von Mises for the model was between 50 to 178 times higher than the nominal contact stress. Comparatively, PLA cage exhibited lower maximal stress value and the stresses was predicted to spread largely on the lateral part of the implant. Thus, lowering the risk of vertebral-endplate deformation and risk of subsidence failure at cage edge proximity.

\section{Conclusion}

This study presents a FEM of PLIF spinal cage implanted bilaterally between L4 and L5 vertebral bone. It 
was clear that spinal cage implant will trigger the cage subsidence at cage-endplate surface. In comparison to PEEK, PLA cages exhibited lower maximal stress at cage-endplate, making the failure risk lesser. Although, the production of high von Mises stress at cage edges is inevitable for PEEK and PLA, the stress distribution in PLA cage was better and the maximum von Mises stress was approximately 3 times lower than PEEK cage. Before confirming the degradation rate of PLA cage in vivo or in vitro, further experiments should be done to comprehend the mechanical properties of PLA cage structure made using the rapid prototyping method.

\section{Acknowledgment}

This work was made possible by the data and advice contributed by the Department of Orthopedic Surgery, Juntendo University, School of Medicine, Japan. The authors thank Hiromitsu Takano and Ikuho Yonezawa for their assistance. Finally, this work was supported by JSPS KAKENHI Grant Number JP15K13836.

\section{References}

[1] Fingar, K. R., Stocks, C., Weiss, A. J., \& Steiner, C. A. (2014). Most frequent operating room procedures performed in u.s. hospitals. HCUP Statistical Brief , 186, 1-15.

[2] Fritzell, P., Berg, S., Borgström, F., Tullberg, T., \& Tropp, H. (2011). Cost effectiveness of disc prosthesis versus lumbar fusion in patients with chronic low back pain: Randomized controlled trial with 2-year follow-up. European Spine Journal, 20(7), 1001-1011.

[3] McKenna, P. J., Freeman, B. J. C., Mulholland, R. C., Grevitt, M. P., Webb, J. K., \& Mehdian, S. H. (2005). A prospective, randomised controlled trial of femoral ring allograft versus a titanium cage in circumferential lumbar spinal fusion with minimum 2-year clinical results. European Spine Journal, 14(8), 727-737.

[4] Vaccaro, A. R. (2002). Spinal applications of bioabsorbable implants. Journal of Neurosurgery: Spine, (4) $97,407-412$.

[5] Dijk, M. V., Smit, T. H., Sugihara, S., Burger, E. H., \& Wuisman, P. I. (2002). The effect of cage stiffness on the rate of lumbar interbody fusion: an in vivo model using poly(l-lactic Acid) and titanium cages. SPINE, 27(7), 682-688,.

[6] Kanayama, M., Cunningham, B. W., Haggerty, C. J., Abumi, K., Kaneda, K., \& McAfee, P. C. (2000). In vitro biomechanical investigation of the stability and stress-shielding effect of lumbar interbody fusion devices. Journal of Neurosurgery:Spine, (2)93, 259-265.

[7] Russias, J., Saiz, E., Nalla, R. K., Gryn, K., Ritchie, R. O., \& Tomsia, A. P. (2006). Fabrication and mechanical properties of PLA/HA composites: A study of in vitro degradation. Materials Science and Engineering: $C$, 26, 1289-1295.

[8] Wuisman, P. I., Dijk, M. V., \& Smit, T. H. (2002). Resorbable cages for spinal fusion: an experimental goat model. Journal of Neurosurgery: Spine, (4)97, 433-439.

[9] Yeong, W. Y., Chua, C. K., Leong, K. F., \& Chandrasekaran, M. (2004). Rapid prototyping in tissue engineering: challenges and potential. TRENDS in Biotechnology, 22(12), 643-52.

[10] Senatov, F. S., Niaza, K. V., Zadorozhnyy, M. Y., Maksimkin, A. V., Kaloshkin, S. D., \& Estrin, Y. Z. (2016). Mechanical properties and shape memory effect of 3D-printed PLA-based porous scaffolds. Journal of the Mechanical Behavior of Biomedical Materials, 57.

[11] Natarajan, R. N., Williams, J. R., \& Andersson, G. B. J. (2006). Modeling changes in intervertebral disc mechanics with degeneration. The Journal of bone and joint surgery. American volume, 88(2), 36-40.

[12] Keyak, J. H., Rossi, S. A., Jones, K. A., \& Skinner, H. B. (1998). Prediction of femoral fracture load using 
automated finite element modeling. Journal of Biomechanics, 31, 125-133.

[13] Mazlan, M. H., Todo, M., Takano, H., \& Yonezawa, I. (2014). Finite element analysis of osteoporotic vertebrae with first lumbar (11) vertebral compression fracture. International Journal of Applied Physics and Mathematics, 4(4), 267-274.

[14] Tawara, D., Noro, K., Tsujikami, T., Okamoto, Y.,\& Murakami, H. (2014). Nonlinear mechanical analysis of posterior spinal instrumentation for osteoporotic vertebra: Effects of mechanical properties of the rod on the failure risks around the screw. Journal of Biomechanical Science and Engineering, 9(2), 1-13.

[15] Adam, C., Pearcy, M., \& McCombe, P. (2003). Stress analysis of interbody fusion - finite element modelling of intervertebral implant and vertebral body. Clinical Biomechanics, 18(4), 265-272.

[16] Mazlan, M. H., Todo, M., Takano, H., \& Yonezawa, I. (2016). Effect of cage insertion orientation on stress profiles and subsidence phenomenon in posterior lumbar interbody fusion. Journal of Medical and Bioengineering, 5(2), 1-6,

[17] Nassau, C. J., Litofsky, N. S., \& Lin, Y. (2012). Analysis of spinal lumbar interbody fusion cage subsidence using Taguchi method, finite element analysis, and artificial neural network. Frontiers of Mechanical Engineering, 7(3), 247-255.

[18] Tsuang, Y. H., Chiang, Y. F., Hung, C. Y., Wei, H. W., Huang, C. H., \& Cheng, C. K. (2009). Comparison of cage application modality in posterior lumbar interbody fusion with posterior instrumentation-A finite element study. Medical Engineering and Physics, 31(5), 565-570.

[19] Bessho, M., Ohnishi, I., Matsuyama, J., Matsumoto, T., Imai, K., \& Nakamura, K. (2007). Prediction of strength and strain of the proximal femur by a CT-based finite element method. Journal of Biomechanics, 40, 1745-1753.

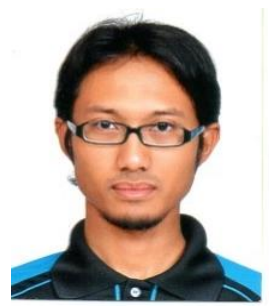

Muhammad Hilmi Jalil was born in Johor, Malaysia. He obtained his bachelor degree of mechanical engineering and the master of applied medical engineering sciences from the Yamaguchi University, Japan. Currently, he is pursuing his PhD at the Kyushu University, Japan in the biomaterial field. He is receiving a fellowship from Faculty of Mechanical Engineering, Universiti Malaysia Pahang, Malaysia.

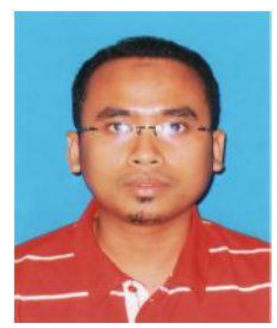

Muhammad Hazli Mazlan was born in Johor, Malaysia, and obtained his bachelor degree of electrical engineering from Kolej Universiti Tun Hussein Onn and the master of biomedical engineering from University of Malaya, Malaysia. He had graduated from the Kyushu University with $\mathrm{PhD}$. He is a lecturer at Faculty of Electrical and Electronic Engineering, Universiti Tun Hussein Onn Malaysia and involved in medical electronics research group.

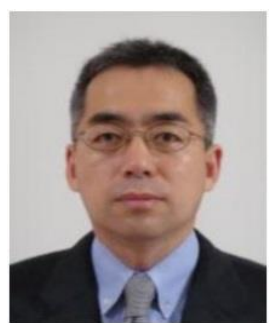

Mitsugu Todo obtained his bachelor and master's degrees of engineering from Kyushu University, Japan, and PhD from The Ohio State University, the United States of America. $\mathrm{He}$ is currently an associate professor of Research Institute for Applied Mechanics, Kyushu University, and working on biomaterials for osteochondral tissue engineering and biomechanics of orthopedic implant. 